\title{
Prevenção e controle de resistência aos antimicrobianos na Atenção Primária à Saúde: evidências para políticas
}

\author{
Prevention and control of antimicrobial resistance \\ in Primary Health Care: evidence for policies
}

Bruna Carolina de Araújo (https://orcid.org/0000-0002-6280-9994) ${ }^{1}$

Roberta Crevelário de Melo (https://orcid.org/0000-0002-2698-9211) ${ }^{1}$

Maritsa Carla de Bortoli (https://orcid.org/0000-0001-8236-7233) ${ }^{1}$

José Ruben de Alcântara Bonfim (https://orcid.org/0000-0002-8518-0615) ${ }^{1}$

Tereza Setsuko Toma (https://orcid.org/0000-0001-9531-9951) ${ }^{1}$
${ }^{1}$ Instituto de Saúde, Secretaria de Estado da Saúde de São Paulo. R.

Santo Antônio 590, Bela

Vista. 01314-000 São

Paulo SP Brasil.

brucarujo@gmail.com

\begin{abstract}
Antimicrobial resistance is a global problem that puts the population's health at risk. This paper aims to identify and evaluate strategies for the prevention and control of antimicrobial resistance, and barriers to their implementation in Primary Health Care (PHC) services. We developed an evidence brief for policies. The search for evidence occurred in 13 databases from November to December 2018. A deliberative dialogue was performed to validate the results and we identified barriers and facilitators to implementing the strategies. The 13 systematic reviews included evidenced that the interventions focused on education, use of electronic systems and biomarkers reduced antimicrobial consumption and prescription. User/caregiver's expectation to receive antibiotic prescriptions was the main obstacle to implementing strategies, while education actions involving health professionals were facilitators. The rational use of medications in the PHC services is crucial to prevent antimicrobial resistance to antibiotics. The interventions identified in this study can be implemented alone or combined, according to local context.
\end{abstract}

Key words Drug microbial resistance, Antimicrobial stewardship, Primary Health Care, Evidence-informed policy
Resumo A resistência aos antimicrobianos é um problema mundial que põe em risco a segurança da saúde da população. O objetivo deste artigo é identificar e avaliar estratégias para prevenção e controle de resistência microbiana, bem como barreiras para sua implementação em serviços de Atenção Primária à Saúde (APS). Realizou-se uma sintese de evidências para politicas. As buscas de evidencias foram realizadas entre novembro/dezembro de 2018, em 13 bases de dados. Um diálogo deliberativo foi realizado para validação dos resultados e levantamento de barreiras e facilitadores para implementação das estratégias. As 13 revisões sistemáticas incluídas mostraram que intervenções com foco em educação, uso de sistemas eletrônicos e biomarcadores reduziram o consumo e prescrição de antimicrobianos. É um obstáculo à implementação a expectativa de usuários/cuidadores em receber prescrição de antibióticos, e são facilitadores as ações educativas que envolvem profissionais de saúde. O uso racional de medicamentos se impõe na APS com vistas à prevenção da resistência dos microrganismos aos antibióticos. As intervenções identificadas neste estudo podem ser implementadas isoladamente ou em conjunto, conforme o contexto local.

Palavras-chave Resistência microbiana a medicamentos, Gestão de antimicrobianos, Atenção Primária à Saúde, Política informada por evidências 


\section{Introdução}

A resistência aos antimicrobianos tornou-se uma ameaça à saúde pública em todo o mundo ${ }^{1}$ e exige respostas nos níveis local, nacional e global ${ }^{2}$, pois como consequência muitos tratamentos têm se tornado ineficazes ${ }^{3}$. É um fenômeno de caráter multifatorial, podendo ocorrer naturalmente por mutação bacteriana, ou ser decorrente do uso inadequado de antibióticos na criação de animais e na produção de alimentos ${ }^{4}$, bem como na atenção à saúde de humanos ${ }^{5}$.

No setor saúde, há grande preocupação com a resistência microbiana em hospitais ${ }^{6}$, particularmente no cuidado a pacientes em unidades de terapia intensiva ${ }^{7}$. No entanto, sabe-se que na Atenção Primária à Saúde (APS) os antimicrobianos são prescritos para situações em que clinicamente não são indicados tais como resfriado comum, dor de garganta, otite média aguda, conjuntivite infecciosa aguda, bronquite e sinusite aguda, causadas por agentes virais ${ }^{8}$. Os consultórios dentários, por sua vez, respondem por $7 \%$ a $10 \%$ de antibióticos prescritos no mundo, sendo que $80 \%$ dessas prescrições podem ser inadequadas ${ }^{9}$. Em países de baixa renda, as prescrições desnecessárias de antibióticos também podem ser influenciadas por situações de incerteza do diagnóstico, em decorrência da dificuldade de acesso a exames complementares. Outro fator que influi nesse tipo de prescrição desnecessária é a falta de acompanhamento de longo prazo de pacientes que apresentam queixas pontuais e não retornam para consulta, não sendo possível avaliar se a conduta terapêutica foi adequada ${ }^{10}$.

Entre 2000 e 2015, o consumo mundial de antibióticos aumentou em $65 \%$, principalmente em países de baixa e média renda ${ }^{5,11}$. Estima-se que aproximadamente $80 \%$ das prescrições de antibióticos ocorram na APS e 90\% do seu uso na comunidade ${ }^{11}$. Um estudo brasileiro apontou que o consumo de antimicrobianos pode ser o mesmo quando se compara a atenção hospitalar com a APS. A exemplo disto, em Teresina-PI, no período de junho de 2015 a julho de 2016 a demanda de amoxicilina (500 mg) correspondeu a $75 \%$ da dispensação de medicamentos dos Centros de Atenção Psicossocial e 47\% da dispensação de medicamentos nas Unidades Básicas de Saúde ${ }^{12}$.

Os microrganismos resistentes a medicamentos, segundo dados da Organização Mundial da Saúde (OMS), são principalmente bactérias gram-negativas resistentes a múltiplos antibióticos e outras bactérias cada vez mais resistentes aos fármacos e que provocam doenças comuns: Enterococcus faecium, resistente à vancomicina; Staphylococcus aureus, resistente à meticilina, com sensibilidade intermediária e resistente à vancomicina; Helicobacter pylori, resistente à claritromicina; Campylobacter ssp. e Salmonellae, resistentes às fluoroquinolonas; Neisseria gonorrhoeae, resistente à cefalosporina e às fluoroquinolonas; Streptococcus pneumoniae, sem sensibilidade à penicilina; Haemophilus influenzae, resistente à ampicilina; Shigella spp., resistente às fluoroquinolonas ${ }^{13}$.

No Brasil, os dados nacionais sobre os principais microrganismos encontrados na APS são incipientes, uma vez que não existe um sistema de vigilância para controle de prescrições de antimicrobianos nesse setor ${ }^{12}$. A necessidade de enfrentar esse problema levou ao desenvolvimento de algumas estratégias por órgãos das Nações Unidas, que orientam a adoção de iniciativa em diversos países, como por exemplo, em 2015 “O Plano de Ação Global sobre Resistência Antimicrobiana", aprovado pela $68^{\circ}$ Assembleia Mundial da Saúde para garantir acesso e uso adequado de medicamentos de qualidade para tratar doenças infectantes ${ }^{14}$.

Além disso, existem redes de vigilância da resistência aos antimicrobianos na Europa (European Antimicrobial Resistance Surveillance Network - EARS-Net), Ásia Central e Europa Oriental (Central Asian and Eastern European Surveillance of Antimicrobial Resistance - CAESAR) e América Latina (Red Latinoamericana de Vigilancia de la Resistencia a los Antimicrobianos - ReLAVRA) ${ }^{15}$.

Em nosso país, desde a década de 1970 várias medidas têm sido propostas, incluindo legislações para lidar com a resistência aos antimicrobianos. Em 2017, com base em pactos nacionais e internacionais, a Agência Nacional de Vigilância Sanitária elaborou o "Plano de Ação da Vigilância Sanitária em Resistência aos Antimicrobianos" que abrange estratégias de enfrentamento ao problema na área de saúde pública ${ }^{16}$. Essas estratégias têm o propósito de promover o uso adequado de antimicrobianos e, consequentemente, favorecer a diminuição da resistência microbiana em serviços de saúde com foco na segurança do paciente $^{17}$.

Considerando a relevância da contribuição da APS para esse problema, o artigo aborda as evidências sobre efetividade de estratégias para prevenção e controle da resistência aos antimicrobianos, além de barreiras e facilitadores para sua implementação neste âmbito da atenção à saúde. 


\section{Método}

Este estudo faz parte de um projeto mais abrangente, que identificou opções para lidar com o problema da resistência microbiana em hospitais e na APS. Ele foi realizado conforme a metodologia preconizada pelas ferramentas SUPPORT (SUPporting POlicy relevant Reviews and Trials) que orientam a elaboração de síntese de evidências para apoiar a tomada de decisão em saúde, incluindo as fases de definição de um problema prioritário de saúde, busca e elegibilidade de evidências científicas, formulação de opções para abordar o problema, considerações sobre implementação e equidade e realização de diálogo deliberativo com representantes de atores sociais interessados no problema ${ }^{18}$. Uma síntese de evidências para políticas (policy brief) é desenvolvida, preferencialmente, a partir de resultados de revisões sistemáticas e tem o propósito de atender a uma demanda da gestão do sistema de saúde ${ }^{19}$.

As buscas por evidências de revisões sistemáticas sobre intervenções para políticas foram realizadas com base no problema da resistência antimicrobiana em serviços de saúde. Ocorreram em datas limites diferentes em novembro e dezembro de 2018, nas seguintes bases de dados: Portal Regional da BVS, Medline via PubMed, Health Systems Evidence, PDQ Evidence, Center for Reviews and Dissemination, Embase, Scopus, Web Of Science, Cochrane Collaboration, Proquest dissertations and theses global, SisREBRATS, Biblioteca Digital Brasileira de Teses e Dissertações e Joanna Briggs Institute. Os termos utilizados nas buscas foram "drug resistance", "antimicrobial resistance", "antibiotic resistance", "antimicrobial stewardship" (termo MeSH e entry terms "Stewardship, Antimicrobial", "Antibiotic Stewardship" e "Stewardship, Antibiotic"), "drug resistance, microbial", "'resistência aos antimicrobianos", "resistência microbiana", "resistência microbiana a medicamentos", "farmacorresistência microbiana", "resistência a antibióticos", "resistência microbiana a antibióticos" e "resistência microbiana a drogas". As estratégias de buscas de cada fonte de informação encontram-se no Quadro 1. A síntese de evidências incluiu revisões sistemáticas com intervenções de enfrentamento da resistência aos antimicrobianos em serviços de saúde publicadas em inglês, espanhol e português. Neste artigo são apresentados resultados de estudos realizados em serviços de APS e ambulatórios. Foram excluídos estudos que abordavam diretrizes sobre tratamentos farmacológicos ou que foram realizados na comunidade, em hospitais ou em outros tipos de serviços de saúde.
Os processos de seleção, extração e avaliação da qualidade metodológica das revisões sistemáticas foram realizados por duas revisoras (BCA e $\mathrm{RCM}$ ), de forma independente, e as divergências foram sanadas por consenso. A avaliação da qualidade metodológica das revisões sistemáticas foi realizada por BCA e RCM, utilizando-se a ferramenta AMSTAR 2 (Assessment of Multiple Systematic Reviews ${ }^{20}$, e revisada por MCB e TST.

A identificação de possíveis barreiras e facilitadores para a implementação das intervenções propostas foi realizada por meio de dados de literatura e de contribuições de participantes de um diálogo deliberativo realizado em novembro de 2019. Os dados de literatura, acessados a partir de uma busca adicional para complementar informações das revisões sistemáticas foram incluídas no Quadro 2. O diálogo deliberativo foi realizado com o objetivo de validar os resultados da síntese de evidências. Os 23 profissionais participantes do diálogo deliberativo eram pessoas com atuação relevante na área temática, sendo 8 pesquisadores, 7 profissionais de saúde e 8 gestores de saúde. A síntese de evidências foi enviada a todos por correio eletrônico, com um mês de antecedência. O diálogo deliberativo é uma estratégia para a formulação de políticas informadas por evidências científicas, realizada por meio de encontro envolvendo os sujeitos interessados, no qual os participantes tecem prós e contras a respeito de ações propostas a partir de suas experiências de pesquisa, gestão ou assistência ${ }^{21,22}$.

Esse processo gerou um documento com quatro opções para tratar do problema da resistência a antimicrobianos, dos quais três eram relativos a hospitais ${ }^{23}$. Neste artigo será apresentada a opção que aborda intervenções efetivas para lidar com o problema na APS e ambulatórios.

\section{Resultados}

De 5.462 estudos identificados, após o processo de seleção foram analisadas 13 revisões sistemáticas sobre estratégias para prevenção e controle da resistência aos antimicrobianos na APS e ambulatório. Na Figura 1, o fluxograma mostra os detalhes do processo de seleção. As características das revisões sistemáticas de qualidade metodológica alta e as de qualidade baixa e criticamente baixa encontram-se, respectivamente nos Quadros 3 e 4.

Quanto à qualidade metodológica (Figura 2), cinco revisões sistemáticas foram avaliadas como de alta qualidade ${ }^{25-29}$, três de qualidade baixa ${ }^{17,30,31}$ e cinco de qualidade criticamente baixa ${ }^{6,32-35}$. 
Quadro 1. Estratégias de busca.

\begin{tabular}{|c|c|c|c|}
\hline Base de dados & Data & Estratégia de busca & Resultado \\
\hline PubMed & $14 / 11 / 2018$ & 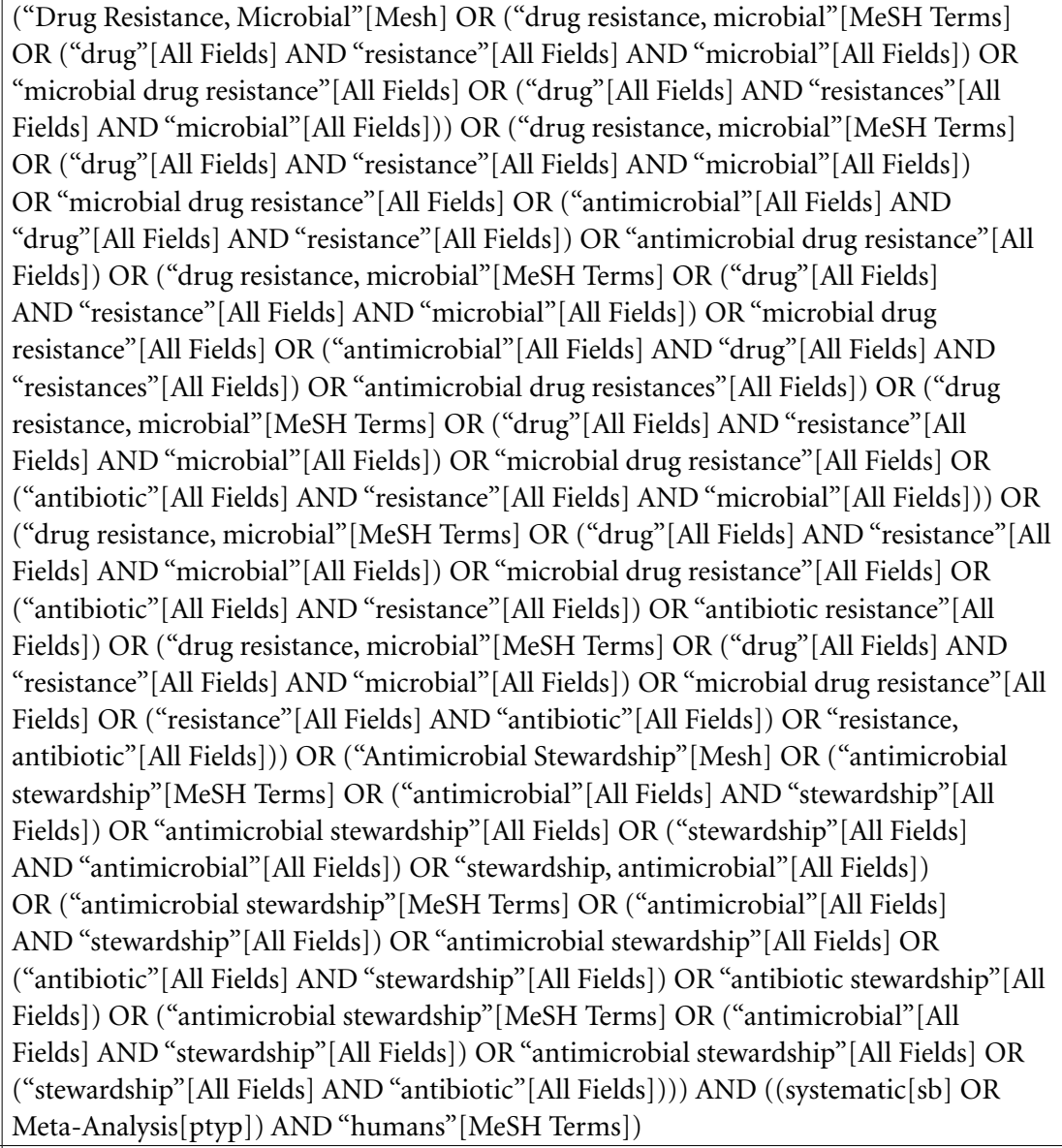 & 2.203 \\
\hline Embase & $14 / 11 / 2018$ & $\begin{array}{l}\text { (('systematic review'/exp OR 'systematic review') AND [embase]/lim OR (('meta } \\
\text { analysis'/exp OR 'meta analysis') AND [embase]/lim)) AND (('antibiotic resistance'/exp } \\
\text { OR 'antibiotic resistance') AND [embase]/lim OR (('antimicrobial stewardship'/exp OR } \\
\text { 'antimicrobial stewardship') AND [embase]/lim)) AND [embase]/lim NOT ([embase]/ } \\
\text { lim AND [medline]/lim) }\end{array}$ & 645 \\
\hline $\begin{array}{l}\text { Portal } \\
\text { regional da } \\
\text { BVS }\end{array}$ & $02 / 11 / 2018$ & 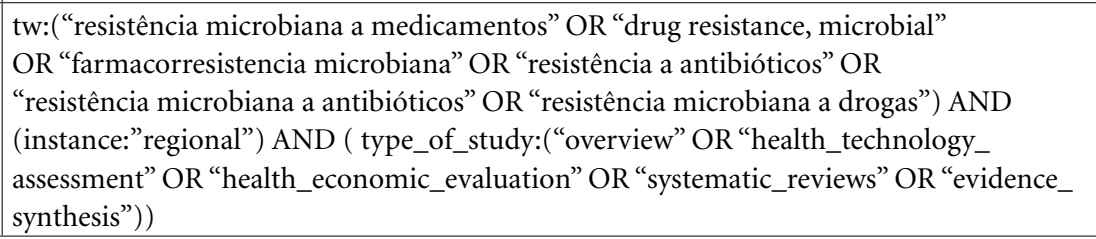 & 39 \\
\hline
\end{tabular}

continua

As estratégias encontradas nestas revisões sistemáticas dividem-se em únicas ou combinadas, sendo que ambas apresentam intervenções ou programas para gerenciamento do uso de antibióticos (antimicrobial stewardship programme) utilizados na APS $^{25-29,31,32,34,35}$ em clínicas pediátricas $^{31}$, ambulatórios em geral ${ }^{6,17,31,32,34}$ e ambulatórios pediátricos ${ }^{33}$. As ações foram conduzidas por profissionais de saúde $e^{6,17,25,26,29-32,34,35}$, médicos ${ }^{28,33}$ e farmacêuticos ${ }^{27}$, tendo como audiência mé$\operatorname{dicos}^{6,25,26,28,30,31,33-35}$, farmacêuticos ${ }^{31,35}$, cuidadores $^{28,31-33}$ e pacientes ${ }^{17,28,29,33}$.

As treze revisões sistemáticas analisadas envolveram pacientes em geral ${ }^{25,34}$, crianças/adolescentes $^{17,25,29,31-33}$, população adulta ${ }^{25,26,29}$, prescritores $^{30,31,35}$, profissionais de saúde especialmente farmacêuticos e enfermeiros ${ }^{35}$, dentistas ${ }^{27}$, cuidadores $^{28,33}$, ou não especificaram a população $0^{6,34}$. 
Quadro 1. Estratégias de busca.

\begin{tabular}{|c|c|c|c|}
\hline Base de dados & Data & Estratégia de busca & Resultado \\
\hline $\begin{array}{l}\text { CRD - } \\
\text { Center for } \\
\text { Reviews and } \\
\text { Dissemination }\end{array}$ & $02 / 11 / 2018$ & (Drug Resistance) IN NHSEED - estudos de avaliação econômica & 150 \\
\hline \begin{tabular}{l|} 
HSE - Health \\
Systems \\
Evidence
\end{tabular} & $02 / 11 / 2018$ & "drug resistance" OR "antimicrobial resistance" & 41 \\
\hline PDQ-evidence & $02 / 11 / 2018$ & "drug resistance" OR “antimicrobial resistance" & 10 \\
\hline Scopus & $14 / 11 / 2018$ & $\begin{array}{l}\text { (TITLE-ABS-KEY ("antibiotic resistance") OR TITLE-ABS-KEY ( “antimicrobial } \\
\text { stewardship") ) AND ( TITLE-ABS-KEY ("systematic review”) OR TITLE-ABS-KEY ( } \\
\text { "meta analysis") ) }\end{array}$ & 2.000 \\
\hline $\begin{array}{l}\text { Web Of } \\
\text { Science }\end{array}$ & $14 / 11 / 2018$ & $\begin{array}{l}\text { TS=(“antibiotic resistance”) OR TI=(“antimicrobial stewardship”) } \\
\text { Índices=SCI-EXPANDED, SSCI, A\&HCI, CPCI-S, CPCI-SSH, ESCI Tempo } \\
\text { estipulado=Todos os anos AND TS=(“systematic review”) OR TI=(“meta analysis”) } \\
\text { Índices=SCI-EXPANDED, SSCI, A\&HCI, CPCI-S, CPCI-SSH, ESCI Tempo } \\
\text { estipulado=Todos os anos }\end{array}$ & 330 \\
\hline $\begin{array}{l}\text { Cochrane } \\
\text { Collaboration }\end{array}$ & $14 / 11 / 2018$ & "antibiotic resistance" OR "antimicrobial stewardship" & 19 \\
\hline $\begin{array}{l}\text { Proquest } \\
\text { dissertations } \\
\text { and theses } \\
\text { global }\end{array}$ & 27/11/2018 & $\begin{array}{l}\text { "antibiotic resistance" OR "antimicrobial stewardship" AND "systematic review" OR } \\
\text { "meta analysis" }\end{array}$ & 13 \\
\hline $\begin{array}{l}\text { BDTD } \\
\text {-Biblioteca } \\
\text { Digital } \\
\text { Brasileira } \\
\text { de Teses e } \\
\text { Dissertações }\end{array}$ & $10 / 12 / 2018$ & $\begin{array}{l}\text { "antibiotic resistance" OR "antimicrobial stewardship" E Todos os campos:"systematic } \\
\text { review" OR "meta analysis" }\end{array}$ & 2 \\
\hline SISREBRATS & $07 / 12 / 2018$ & $\begin{array}{l}\text { 'resistência microbiana' OR 'antimicrobial stewardship' OR 'antibiotic resistance' OR } \\
\text { 'resistência aos antimicrobianos' }\end{array}$ & 3 \\
\hline
\end{tabular}

Fonte: Autores.

Quadro 2. Estratégias de busca de estudos de implementação.

\begin{tabular}{|c|c|c|c|}
\hline Base de dados & Data & Estratégia de busca & Resultado \\
\hline PubMed & $02 / 06 / 2019$ & 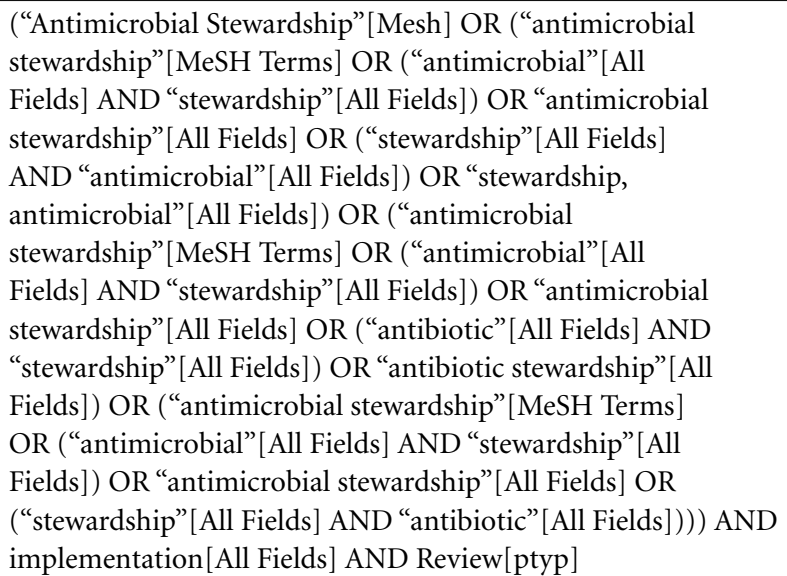 & 150 \\
\hline $\begin{array}{l}\text { CRD - } \\
\text { Center for } \\
\text { Reviews and } \\
\text { Dissemination }\end{array}$ & $06 / 08 / 2019$ & "stewardship" & 5 \\
\hline
\end{tabular}

Fonte: Autores. 


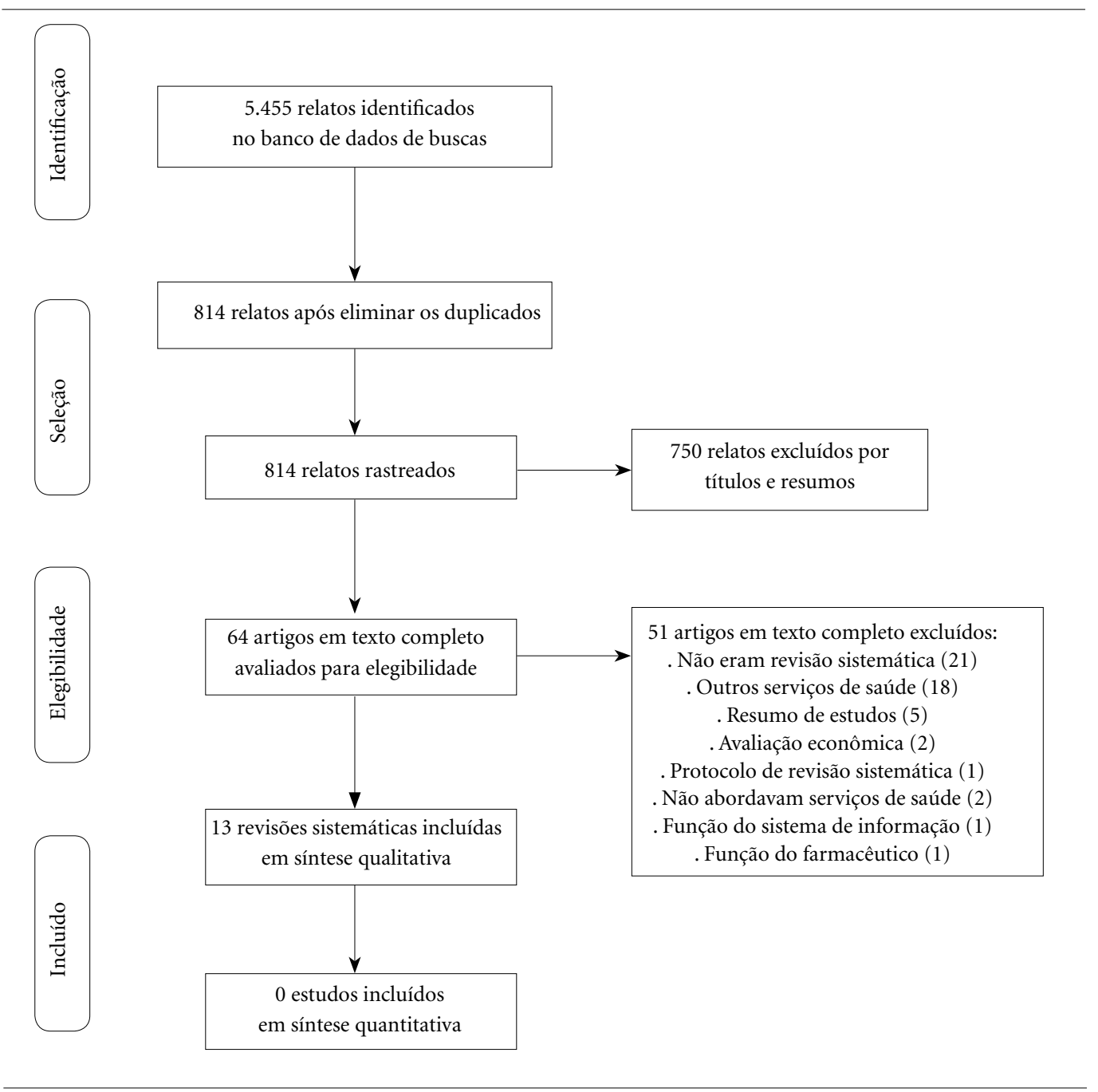

Figura 1. Fluxograma do processo de seleção dos artigos.

Fonte: Autores, adaptado da recomendação PRISMA ${ }^{24}$.

Os estudos primários incluídos nessas revisões sistemáticas foram conduzidos em países de baixa renda ${ }^{17,27}$, média baixa renda ${ }^{30,35}$, média alta renda ${ }^{25,26,29-31,34}$ e alta renda ${ }^{17,25-31}$. Estes estudos apresentaram diversos períodos de duração e acompanhamento, variando de um mês ${ }^{31}$ até doze meses $^{27}$.

Os resultados das intervenções analisadas nas revisões sistemáticas são apresentados a seguir, mostrando desfechos secundários à redução ou prevenção da resistência antimicrobiana, como redução na prescrição e no uso de antibióticos, redução no tempo de tratamento e melhora na adesão às diretrizes sobre uso apropriado de antimicrobianos.
Houve redução no uso de antibióticos com estratégias de decisão compartilhada ${ }^{26}$, teste de proteína C-reativa ${ }^{25}$, prescrições $\operatorname{tardias}^{32}$, uso de sistemas eletrônicos ${ }^{6}$ e intervenções educativas $^{6,28,33,35}$.

Já em relação à prescrição de antibióticos, houve redução com o uso de sistemas eletrôni$\cos ^{6,17}$, biomarcadores ${ }^{17}$, ações educativas ${ }^{31}$, tomada de decisão compartilhada, uso de diretrizes para prescrição, feedback individual do provedor e treinamento de habilidades de comunicação "centradas no paciente"17, sem aumentar o número de retornos a consultas ou diminuir a satisfação do paciente com a consulta. Intervenções combinadas reduziram a prescrição de antibióti- 
Quadro 3. Características das revisões sistemáticas, de qualidade metodológica alta, incluídas na formulação da opção para políticas de prevenção e controle de resistência aos antimicrobianos na APS e ambulatórios.

\begin{tabular}{|c|c|c|c|c|}
\hline $\begin{array}{l}\text { Autor, } \\
\text { Ano }\end{array}$ & $\begin{array}{l}\text { População/ } \\
\text { Local }\end{array}$ & $\begin{array}{l}\text { No de estudos } \\
\text { primários } \\
\text { incluídos/Tipo } \\
\text { de estudo }\end{array}$ & $\begin{array}{c}\text { Principais elementos das } \\
\text { intervenções }\end{array}$ & Desfechos relatados \\
\hline $\begin{array}{l}\text { Aabenhus } \\
\text { et al., } \\
2014^{25}\end{array}$ & $\begin{array}{l}3.284 \text { adultos } \\
139 \text { crianças } \\
\text { APS }\end{array}$ & $\begin{array}{l}3 \text { ECR-cluster } \\
3 \text { ECR }\end{array}$ & Teste de proteína C-reativa & $\begin{array}{l}\text { Redução do uso de antibióticos } \\
\text { - Antibióticos prescritos na consulta } \\
\text { (6 ECR, } 3.284 \text { participantes, RR=0,78, IC } 95 \% \\
0,66 \text { a } 0,92, \mathrm{I}^{2}=68 \% \text { ) } \\
\text { - Antibióticos prescritos em } 28 \text { dias (com } \\
\text { amostra modificada) } \\
\text { (4 ECR, } 708 \text { participantes, RR=0,80, IC95\% } \\
0,67 \text { a } 0,96, \mathrm{I}^{2}=40 \% \text { ) }\end{array}$ \\
\hline $\begin{array}{l}\text { Coxeter et } \\
\text { al., } \\
2015^{26}\end{array}$ & $\begin{array}{l}1.100 \text { médicos } \\
492.000 \\
\text { pacientes } \\
\text { Ambulatório } \\
\text { e APS }\end{array}$ & $\begin{array}{l}9 \text { ECR-cluster } \\
1 \text { ECR }\end{array}$ & $\begin{array}{l}\text { Materiais impressos, grupos } \\
\text { de discussão sobre as diretrizes } \\
\text { e cursos de aperfeiçoamento } \\
\text { aos prescritores; intervenção } \\
\text { educacional complexa, liderada } \\
\text { por pares; treinamento de } \\
\text { comunicação centrada no } \\
\text { paciente; treinamento em } \\
\text { habilidades de comunicação; } \\
\text { folheto interativo sobre } \\
\text { infecções do trato respiratório } \\
\text { em crianças para uso durante } \\
\text { a consulta e como um recurso } \\
\text { para levar para casa }\end{array}$ & $\begin{array}{l}\text { Redução do uso de antibióticos } \\
\text { - Antibióticos prescritos ou dispensados ( } 6 \\
\text { semanas ou menos) } \\
\text { ( } 8 \text { ECR, } 10.172 \text { participantes, } \mathrm{RR}=0,61, \mathrm{IC} 95 \% \\
\left.0,55 \text { a } 0,65, \mathrm{I}^{2}=0 \%\right) \\
\text { - Antibióticos prescritos ou dispensado ( } 12 \\
\text { meses ou mais) } \\
(3 \mathrm{ECR}, 481.588 \text { participantes, } \mathrm{RR}=0,74, \\
\left.\text { IC95\% } 0,49 \text { a } 1,11, \mathrm{I}^{2}=89 \%\right)\end{array}$ \\
\hline $\begin{array}{l}\text { Löffler e } \\
\text { Böhmer, } \\
2017^{27}\end{array}$ & $\begin{array}{l}1.307 \\
\text { dentistas } \\
\text { APS }\end{array}$ & $\begin{array}{l}3 \text { ECR-cluster } \\
2 \text { ECR } \\
4 \text { ECNR }\end{array}$ & $\begin{array}{l}\text { Auditorias clínicas, visitas } \\
\text { educacionais, feedback, } \\
\text { educação do paciente e } \\
\text { treinamento em comunicação } \\
\text { para profissionais }\end{array}$ & $\begin{array}{l}\text { Redução da prescrição de antibióticos } \\
\text { - } 9 \text { estudos mostraram a redução das } \\
\text { prescrições antimicrobianas que variaram } \\
\text { de } 42,5 \% \text { a } 89,5 \% \text {, sendo que estudos com } \\
\text { populações menores apresentaram maiores } \\
\text { taxas de redução }\end{array}$ \\
\hline $\begin{array}{l}\text { O'Sullivan } \\
\text { et al., } \\
2016^{28}\end{array}$ & $\begin{array}{l}558 \text { crianças } \\
269 \text { médicos } \\
\text { APS }\end{array}$ & $2 \mathrm{ECR}$ & $\begin{array}{l}\text { Informações escritas para } \\
\text { pacientes }\end{array}$ & $\begin{array}{l}\text { Redução do uso de antibióticos } \\
\text { - Antibióticos utilizados pelos pacientes } \\
(1 \mathrm{ECR}, 220 \text { participantes, } \mathrm{RR}=0,53, \mathrm{IC} 95 \% \\
0,35 \text { a } 0,80, \mathrm{I}^{2}=\text { não se aplica) } \\
\text { - Antibióticos prescritos pelos médicos } \\
(2 \mathrm{ECR}, 18.813 \text { participantes, } \mathrm{RR}=0,47, \mathrm{IC} 95 \% \\
0,28 \text { a } 0,78, \mathrm{I}^{2}=\text { não informado) }\end{array}$ \\
\hline $\begin{array}{l}\text { Schuetz et } \\
\text { al., } \\
2017^{29}\end{array}$ & $\begin{array}{l}1.008 \\
\text { participantes } \\
\text { APS, } \\
\text { Departamento } \\
\text { de Emergência } \\
\text { e Hospital }\end{array}$ & $2 \mathrm{ECR}$ & Teste de procalcitonina & $\begin{array}{l}\text { Redução da exposição total aos antibióticos } \\
\text { - Exposição total de antibióticos (dias), } \\
\text { média ( } \pm \mathrm{DP})(1.008 \text { participantes, } \\
\text { intervenção= }=4,6 \pm 4,1, \text { controle }=1,6 \pm 3,2, \mathrm{OR} \\
\text { ajustado=-3,0, IC } 95 \%-3,45 \text { a }-2,58, \mathrm{I}^{2}=\text { não } \\
\text { informado) } \\
\text { Redução do tempo de tratamento } \\
\text { - Duração dos antibióticos (dias), média }( \pm \mathrm{DP}) \\
(1.008 \text { participantes, intervenção=7,3 } \pm 2,5, \\
\text { controle }=7,0 \pm 2,8, \text { OR ajustado=- } 0,52, \mathrm{IC} 95 \% \\
-1,07 \text { a } 0,04 ; \mathrm{I}^{2}=\text { não informado) }\end{array}$ \\
\hline
\end{tabular}

Nota: $\mathrm{APS}=$ atenção primária à saúde; $\mathrm{DP}=$ desvio padrão; $\mathrm{ECR}=$ ensaio clínico randomizado; $\mathrm{ECNR}=$ ensaio clínico não randomizado; $\mathrm{OR}=\mathrm{odd}$ ratio; $\mathrm{RR}=$ risco relativo; $\mathrm{I}^{2}=$ medida de heterogeneidade; $\mathrm{IC} 95 \%=$ intervalo de confiança de $95 \%$.

Fonte: Autores. 


\begin{tabular}{|c|c|c|c|c|}
\hline \multicolumn{5}{|c|}{$\begin{array}{l}\text { Quadro 4. Características das revisões sistemáticas, de qualidade metodológica baixa ou criticamente baixa, incluídas na formulação da } \\
\text { opção para políticas de prevenção e controle de resistência aos antimicrobianos na APS e ambulatórios. }\end{array}$} \\
\hline $\begin{array}{l}\text { Autor, } \\
\text { ano }\end{array}$ & $\begin{array}{l}\text { População/ } \\
\text { Local }\end{array}$ & $\begin{array}{l}\text { No de estudos } \\
\text { primários } \\
\text { incluídos/ } \\
\text { Tipo de } \\
\text { estudo } \\
\end{array}$ & Principais elementos das intervenções & Desfechos relatados \\
\hline $\begin{array}{l}\text { Arnold e } \\
\text { Straus, } \\
2005^{30}\end{array}$ & $\begin{array}{l}9.326 \\
\text { médicos } \\
\text { APS e } \\
\text { Ambulatório }\end{array}$ & $\begin{array}{l}25 \text { ECR } \\
14 \text { ECNR }\end{array}$ & $\begin{array}{l}\text { Reuniões educativas; visitas educacionais } \\
\text { realizadas por especialistas }\end{array}$ & $\begin{array}{l}\text { Redução da prescrição de antibióticos } \\
\text { - } 9 \text { estudos indicaram uma redução nas } \\
\text { prescrições médicas através de reuniões } \\
\text { educativas } \\
\text { - } 8 \text { estudos mostraram redução nas } \\
\text { prescrições de tetraciclina, cefalexina, por } \\
\text { meio de reunião com farmacêuticos }\end{array}$ \\
\hline $\begin{array}{l}\text { Hu et al., } \\
2016^{31}\end{array}$ & $\begin{array}{l}1.707 \\
\text { médicos } \\
14.018 \\
\text { pacientes } \\
97.699 \\
\text { crianças } \\
1.351 \text { pais } \\
\text { APS e CP }\end{array}$ & $\begin{array}{l}7 \text { ECR-cluster } \\
3 \mathrm{ECNR} \\
2 \mathrm{ECR}\end{array}$ & $\begin{array}{l}\text { Para prescritores: apresentação de taxas de } \\
\text { prescrição de antibióticos, atualização ou } \\
\text { reforço das diretrizes nacionais, incentivo } \\
\text { a prescrições tardias treinamento de } \\
\text { habilidades de comunicação entre médico } \\
\text { e pais } \\
\text { Para pais: materiais educacionais impressos, } \\
\text { incluindo folhetos, panfletos ou pôsteres, } \\
\text { meios de comunicação }\end{array}$ & $\begin{array}{l}\text { Redução da prescrição de antibióticos } \\
\text { - Efeito da redução de prescrição de } \\
\text { antibióticos com todas as intervenções (NA, } \\
\left.\text { OR=0,63, IC95\% 0,50 a } 0,81, \mathrm{I}^{2}=66 \%\right) \\
\text { - Efeito das estratégias de intervenção com } \\
\text { os médicos na taxa prescrição de prescrição } \\
\text { de antibióticos (NA, OR=0,65, IC95\% 0,54 a } \\
\left.0,79, \mathrm{I}^{2}=44 \%\right) \\
\text { - Efeitos das estratégias de intervenção com } \\
\text { os pais } \\
\text { - Efeitos na taxa de prescrição de } \\
\text { antibióticos para todas as estratégias (NA, } \\
\left.\text { OR=0,55, IC95\% } 0,36 \text { a } 0,84, \mathrm{I}^{2}=36 \%\right)\end{array}$ \\
\hline $\begin{array}{l}\text { Andrews } \\
\text { et al., } \\
2012^{32}\end{array}$ & $\begin{array}{l}771 \text { pais } \\
\text { APS e CPU }\end{array}$ & $\begin{array}{l}3 \mathrm{ECR} \\
1 \mathrm{ECNR}\end{array}$ & $\begin{array}{l}\text { Panfleto e livro acompanhados de } \\
\text { orientação verbal aos pais ou cuidadores }\end{array}$ & $\begin{array}{l}\text { Redução do uso de antibióticos } \\
\text { - Efeito geral das intervenções sobre o uso } \\
\text { de antibióticos prescritos (NA no de ensaios } \\
\text { e de participantes; RR combinado=0,39, } \\
\text { IC95\% } 0,29 \text { a } 0,53, \mathrm{I}^{2}=\text { não informado) }\end{array}$ \\
\hline $\begin{array}{l}\text { Drekonja } \\
\text { et al., } \\
2015^{17}\end{array}$ & $\begin{array}{l}\text { Não } \\
\text { informado } \\
\text { Ambulatório }\end{array}$ & $\begin{array}{l}18 \text { ECR- } \\
\text { cluster } \\
17 \text { ECR } \\
9 \text { ECNR } \\
6 \text { STI }\end{array}$ & $\begin{array}{l}\text { Diretrizes; Regulação para os prescritores; } \\
\text { Teste de proteína C-reativa; Teste } \\
\text { de procalcitonina; Teste de detecção } \\
\text { de antígenos; Auditoria e feedback; } \\
\text { Treinamento de habilidades de } \\
\text { comunicação; Retardo na prescrição; } \\
\text { Sistemas informatizados de suporte à } \\
\text { decisão }\end{array}$ & $\begin{array}{l}\text { Redução da prescrição de antibióticos } \\
\text { - } 9 \text { estudos encontraram diminuição de } \\
\text { prescrições de antibióticos, principalmente } \\
\text { relacionadas a infecções respiratórias e } 6 \\
\text { estudos não encontraram diferenças entre } \\
\text { os grupos }\end{array}$ \\
\hline
\end{tabular}

continua

cos, sobretudo para doenças específicas ${ }^{34}$. Educação médica, de pacientes e do público em locais e formatos variados foram os mais bem-sucedidos na redução da prescrição desnecessária de antibióticos ${ }^{30}$.

Observou-se redução de antibióticos prescritos e melhora na escolha de antibióticos apropriados com ações educativas ${ }^{35}$, e aumento na precisão da prescrição de antibióticos em odontologia por meio de auditorias clínicas, visitas educacionais, feedback, educação do paciente e treinamento em comunicação ${ }^{27}$.
A adesão a diretrizes foi maior com sessões de feedback e de treinamento para a implantação de diretrizes ${ }^{6}$ e ações educativas ${ }^{35}$. Teste de procalcitonina mostrou reduzir significativamente a exposição total aos antibióticos e o tempo de tratamento ${ }^{29}$.

Apenas uma revisão sistemática informou sobre custo-efetividade das intervenções estuda$\mathrm{das}^{30}$. Arnold e Straus ${ }^{30}$ apontaram que diretrizes clínicas, reuniões educativas e auditoria com $f e$ edback podem ser custo-efetivas, na redução da prescrição de antibióticos. 
Quadro 4. Características das revisões sistemáticas, de qualidade metodológica baixa ou criticamente baixa, incluídas na formulação da opção para políticas de prevenção e controle de resistência aos antimicrobianos na APS e ambulatórios.

\begin{tabular}{|c|c|c|c|c|}
\hline $\begin{array}{l}\text { Autor, } \\
\text { ano }\end{array}$ & $\begin{array}{l}\text { População/ } \\
\text { Local }\end{array}$ & $\begin{array}{l}\text { No de estudos } \\
\text { primários } \\
\text { incluídos/ } \\
\text { Tipo de } \\
\text { estudo }\end{array}$ & Principais elementos das intervenções & Desfechos relatados \\
\hline $\begin{array}{l}\text { Keller et } \\
\text { al., } \\
2018^{6}\end{array}$ & $\begin{array}{l}\text { Não } \\
\text { informado } \\
\text { Ambulatório }\end{array}$ & $\begin{array}{l}16 \text { ECR } \\
16 \text { ECR- } \\
\text { cluster } \\
9 \text { quasi- } \\
\text { randomizado }\end{array}$ & $\begin{array}{l}\text { Programa de engenharia de fatores } \\
\text { humanos especializado em saúde }\end{array}$ & $\begin{array}{l}\text { Redução do uso de antibióticos } \\
\text { - } 10 \text { estudos avaliaram que intervenções } \\
\text { baseadas em CDSS e EHR foram eficazes, } \\
\text { principalmente quando o EHR foi } \\
\text { vinculado à justificativa de um prescritor } \\
\text { em recomendar o uso de um agente } \\
\text { antimicrobiano } \\
\text { Redução da prescrição de antibióticos } \\
\text { - } 13 \text { estudos verificaram que intervenções de } \\
\text { auditoria e feedback nas quais houve uma } \\
\text { comparação entre os médicos foram eficazes } \\
\text { na redução de prescrições de antibióticos }\end{array}$ \\
\hline $\begin{array}{l}\text { Patel et } \\
\text { al., } \\
2007^{33}\end{array}$ & $\begin{array}{l}\text { Não } \\
\text { informado } \\
\text { Ambulatório } \\
\text { Pediátrico }\end{array}$ & $\begin{array}{l}9 \text { ECNR } \\
9 \text { STI } \\
7 \text { ECR } \\
2 \text { Coorte }\end{array}$ & $\begin{array}{l}\text { Intervenção restritiva, ações educacionais e } \\
\text { feedback }\end{array}$ & $\begin{array}{l}\text { Redução do uso de antibióticos } \\
\text { - } 8 \text { estudos indicaram que ações } \\
\text { educativas melhoraram a prescrição de } \\
\text { antimicrobianos medida pelo consumo } \\
\text { de antibióticos ou melhora nas taxas de } \\
\text { investigação adequada }\end{array}$ \\
\hline $\begin{array}{l}\text { Ivanovska } \\
\text { e } \\
\text { Holloway, } \\
2013^{34}\end{array}$ & $\begin{array}{l}1.144 .849 \\
\text { casos } \\
\text { APS e } \\
\text { Ambulatório }\end{array}$ & $\begin{array}{l}1 \mathrm{ECR} \\
7 \mathrm{ECNR}\end{array}$ & $\begin{array}{l}\text { Educação do provedor, educação do } \\
\text { usuário, educação e supervisão do provedor, } \\
\text { educação do provedor com estratégias de } \\
\text { processo em grupo - comitê de revisão por } \\
\text { pares e comitê de farmácia, educação de } \\
\text { provedores e usuários com supervisão, e } \\
\text { intervenção multifacetada com estratégias } \\
\text { educacionais, econômicas e lista de } \\
\text { medicamentos essenciais }\end{array}$ & $\begin{array}{l}\text { Redução da prescrição de antibióticos } \\
\text { - } 7 \text { estudos informaram que ações } \\
\text { educativas únicas ou multifacetadas podem } \\
\text { impactar nas prescrições médicas de } \\
\text { antibióticos }\end{array}$ \\
\hline $\begin{array}{l}\text { Roque et } \\
\text { al., } \\
2014^{35}\end{array}$ & $\begin{array}{l}559.280 \\
\text { participantes } \\
\text { APS }\end{array}$ & $\begin{array}{l}26 \text { ECR } \\
10 \text { ECNR } \\
13 \text { pré-pós } \\
\text { teste }\end{array}$ & $\begin{array}{l}\text { Distribuição de formulários, protocolos } \\
\text { e diretrizes, materiais autoinstrucionais, } \\
\text { sessões de grupo, conferências, palestras, } \\
\text { seminários e tutoriais; feedback dos } \\
\text { padrões de prescrição médica ou feedback } \\
\text { do paciente sobre listas específicas de } \\
\text { medicamentos prescritos para ele; visitas } \\
\text { individuais de divulgação; lembretes } \\
\text { no momento da prescrição; sistemas } \\
\text { de tomada de decisão auxiliados por } \\
\text { computador; processo de controle de } \\
\text { forma restritiva; educação do paciente } \\
\text { (panfletos, fitas de vídeo); cursos sobre } \\
\text { testes rápidos, testes rápidos de antígenos } \\
\text { em consultórios; aplicação de regulamentos; } \\
\text { feedback de farmacêuticos e médicos de } \\
\text { doenças infecciosas sobre a prescrição } \\
\text { com recomendações para modificação; } \\
\text { incentivos financeiros }\end{array}$ & $\begin{array}{l}\text { Redução do uso de antibióticos } \\
\text { - } 30 \text { estudos apresentaram diminuição } \\
\text { no uso de antibiótico, com exceção } \\
\text { de feedback de farmacêuticos e médicos } \\
\text { de doenças infecciosas sobre a prescrição } \\
\text { com recomendações para modificação e } \\
\text { incentivos financeiros } \\
\text { Melhora da adesão a diretrizes } \\
\text { - } 18 \text { estudos mostraram que intervenções } \\
\text { educativas e incentivos financeiros } \\
\text { melhoraram a aderência às orientações } \\
\text { de antibióticos de acordo com algoritmos } \\
\text { de diretrizes, incluindo doses e vias de } \\
\text { administração }\end{array}$ \\
\hline
\end{tabular}

Nota: $\mathrm{APS}=$ atenção primária à saúde; $\mathrm{CDSS}=$ sistemas de apoio à decisão clínica; $\mathrm{CP}=$ clínica pediátrica; $\mathrm{CPU}=$ clínica pediátrica universitária; $\mathrm{DEP}=$ departamento

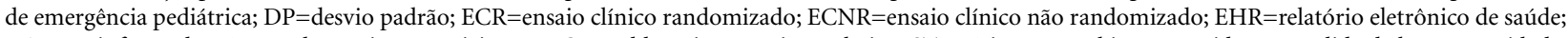
$\mathrm{NA}=$ não informado número de ensaios e participantes; $\mathrm{OR}=$ odds ratio; $\mathrm{RR}=$ risco relativo; $\mathrm{STI}=$ série temporal interrompida; $\mathrm{I}^{2}=$ medida de heterogeneidade; IC $95 \%=$ intervalo de confiança de $95 \%$. 


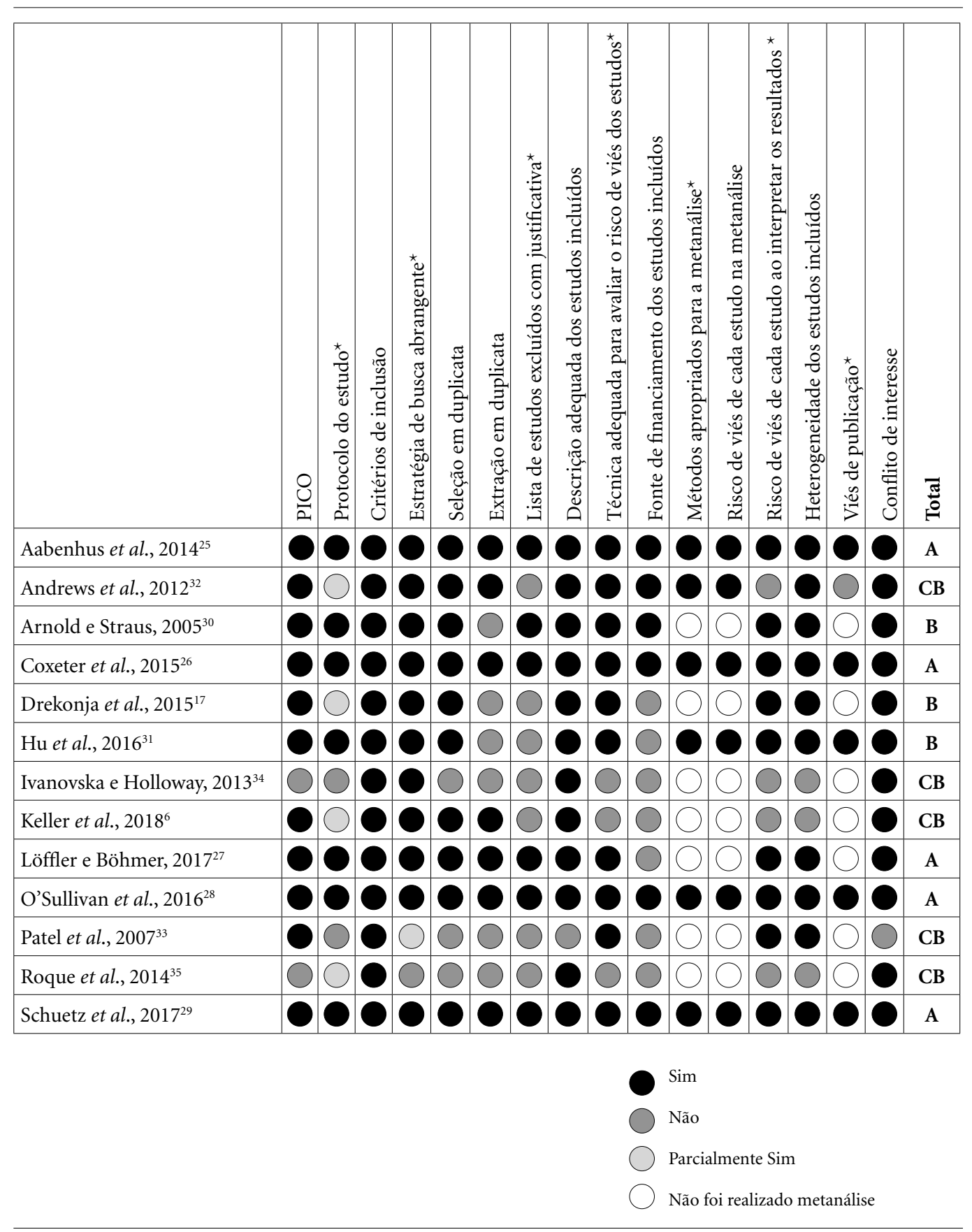

Figura 2. Qualidade metodológica das revisões sistemáticas.

Nota: ${ }^{\star}$ Domínios críticos. A - alto; B - baixo; CB - criticamente baixo.

Fonte: Autores.

As revisões sistemáticas indicaram também incertezas nos resultados de algumas intervenções. Ações educativas podem não apresentar efeito significativo em relação a mudança de comportamento do prescritor ${ }^{30}$, como por exemplo a falta de efetividade de disseminação de materiais educativos impressos (protocolos, orientações e autoinstrução) na redução de prescrições de antibióticos ${ }^{35}$. O uso de lembretes esteve associado ao aumento da adesão a apenas 
algumas recomendações prescritas e demonstrou baixa aceitação do apoio à decisão pelos profissionais ${ }^{17}$. As diretrizes para melhorar a seleção de antimicrobianos mostraram resultados mistos ${ }^{17}$. Informações por escrito também não se mostraram efetivas para reduzir taxas de reconsulta e melhorar a satisfação dos pais ${ }^{28}$.

\section{Considerações sobre a implementação das intervenções na APS e ambulatórios}

A literatura mundial informa sobre algumas barreiras e facilitadores para a implementação das intervenções acima descritas para enfrentar o problema da resistência antimicrobiana na APS e ambulatórios, nos níveis de pacientes/usuários, cuidadores, profissionais de saúde, gestores e sistemas de saúde (Quadro 5). Na busca adicional, foram identificadas 155 publicações. Após o processo de seleção restaram 91 estudos, dos quais 21 estudos (10 revisões de literatura, 7 revisões sistemáticas, e 3 estudos primários) apresentaram resultados referente a APS e ambulatórios.

Foram encontrados mais achados sobre facilitadores de implementação das ações de enfrentamento da resistência a antimicrobianos em ambulatórios do que na APS, especialmente a respeito de intervenções educativas aos profissionais de saúde.

Com relação aos fatores que se configuram como obstáculos à implementação das estratégias para lidar com a resistência microbiana, nos

Quadro 5. Facilitadores e barreiras para a implementação das intervenções na APS em ambulatórios.

\begin{tabular}{|c|c|}
\hline Facilitadores & Barreiras \\
\hline \multicolumn{2}{|c|}{ Usuários } \\
\hline $\begin{array}{l}\text { APS } \\
\text { - Ações educativas para a comunidade e para pais de } \\
\text { pacientes pediátricos }^{31,36} \\
\text { Ambulatórios } \\
\text { - Estratégias para a melhoria da comunicação entre } \\
\text { prescritores e pacientes } \\
\text { - Decisão compartilhada entre paciente e profissional } \\
\text { da saúde no tratamento } \\
\text { 37,38 }\end{array}$ & $\begin{array}{l}\text { APS } \\
\text { - Satisfação de usuários e cuidadores quanto a } \\
\text { prescrição terapêutica }^{31,32} \\
\text { Ambulatórios } \\
\text { - Necessidade do usuário em receber prescrição de } \\
\text { antibióticos }\end{array}$ \\
\hline \multicolumn{2}{|c|}{ Trabalhadores de saúde } \\
\hline $\begin{array}{l}\text { APS } \\
\text { - Melhor organização do processo de trabalho e } \\
\text { envolvimento de representantes dos profissionais } \\
\text { de saúde na tomada de decisão para a escolha das } \\
\text { estratégias que serão implementadas } \text { s }^{17,36,37,39,40} \\
\text { Ambulatórios } \\
\text { - Acesso e a utilização de sistemas eletrônicos }{ }^{17} \\
\text { - Realização de testes rápidos }{ }^{25} \\
\text { - Ações educativas ativas, tempo para profissionais a } \\
\text { executarem dentro do trabalho }{ }^{37,41} \\
\text { - Ações educativas a distância }{ }^{25} \\
\text { - Monitoramento e a avaliação periódicas }{ }^{37,42} \\
\text { - Escolha de uma pessoa-chave que seja respeitada } \\
\text { pelos outros profissionais de saúde }{ }^{42}\end{array}$ & $\begin{array}{l}\text { APS } \\
\text { - Tentativa do prescritor em satisfazer a expectativa do } \\
\text { usuário }^{28,37} \\
\text { - Crenças do conhecimento empírico, medo e } \\
\text { insegurança do prescritor }{ }^{28,32,37,40,44,45,49} \\
\text { - Falta de materiais e recursos adequados e } \\
\text { organização inadequada do trabalho }^{40,45,49} \\
\text { Ambulatórios } \\
\text { - Insegurança dos prescritores na escolha } \\
\text { terapêutica } \\
\text { - Falta de familiaridade dos profissionais com os } \\
\text { sistemas, ou interrupção não prevista no fluxo de } \\
\text { trabalho }{ }^{17,33,37} \\
\text { - Falta de adesão dos profissionais de saúde às } \\
\text { diretrizes clínicas } \\
\text { - Vínculo afetivo entre o médico e paciente influi na } \\
\text { prescrição de antibióticos }^{6}\end{array}$ \\
\hline \multicolumn{2}{|c|}{ Gestores de serviços e sistemas de saúde } \\
\hline $\begin{array}{l}\text { APS } \\
\text { - Inclusão do tema da resistência microbiana na } \\
\text { agenda de prioridades para fortalecimento de } \\
\text { políticas } \\
\text { - Estratratégias de educação e suporte técnico }{ }^{36,43,46,47} \\
\text { Ambulatórios } \\
\text { - Desenvolvimento de diretrizes, seminários e mesas } \\
\text { redondas sobre o tema }{ }^{37,41,42,48}\end{array}$ & $\begin{array}{l}\text { APS } \\
\text { - Problemas de acesso à informação, adaptação } \\
\text { de diretrizes e falta de pactuação de planos e } \\
\text { políticas } \\
\text { Ambu3,45,50 } \\
\text { Ambulatórios } \\
\text { - Disponibilidades de materiais e recursos adequados, } \\
\text { sistemas eletrônicos e organização do trabalho } \\
\text { ineficientes } \\
\text { i,37,48 }\end{array}$ \\
\hline
\end{tabular}

Fonte: Autores. 
dois tipos de serviços de saúde destacaram-se a expectativa de médicos em satisfazer pacientes/ cuidadores com prescrição de medicamentos e a dificuldade de disponibilidade de materiais e recursos para a implementação das ações de enfrentamento da resistência antimicrobiana. No Diálogo Deliberativo, os participantes validaram os achados da literatura e trouxeram algumas contribuições, especialmente relacionadas a barreiras de implementação das ações e agravos do uso inadequado dos antimicrobianos, que são apresentadas a seguir.

Com relação aos usuários do sistema de saúde, um obstáculo é a sua influência na prescrição, uma vez que espera sair da consulta com uma receita de antibiótico. Além disso, fatores como maior tempo de tratamento e falta de acompanhamento do paciente em longo prazo dificultam a prática de uso racional de antimicrobianos. Relatou-se a necessidade de educação em saúde na comunidade sobre o problema da resistência antimicrobiana desde o ensino fundamental. Aspectos de equidade das estratégias, de acordo com o contexto local, devem ser consideradas quando se trata de populações mais vulneráveis, como os imigrantes, pessoas com baixo ou nenhum letramento, refugiados, pessoas em situação de rua ou privadas de liberdade, transexuais e travestis, dependentes químicos, pessoas com tuberculose, hanseníase ou com HIV.

No que se refere aos profissionais de saúde, enfatizou-se a pressão institucional para prescrição de alguns medicamentos com motivação econômica, o que pode interferir na decisão do prescritor para a escolha terapêutica. Também, considerou-se como obstáculos a falta de recursos humanos, deficiência na formação profissional e pouco trabalho interdisciplinar.

Do ponto de vista da organização de serviços, a falta de sequência no acompanhamento do paciente na rede de saúde, sobretudo na APS, com a fragmentação do cuidado, foi considerada uma grande barreira para implementação das ações de enfrentamento da resistência aos antimicrobianos. Destacou-se a dificuldade de acesso a medicamentos de primeira linha e infraestrutura adequada para melhorar a articulação entre os serviços de saúde.

Considerou-se que as políticas e produções acadêmicas disponíveis, em sua maioria, são voltadas para o ambiente hospitalar, negligenciando a APS, outros ambientes como as instituições de longa permanência e outros setores para além da saúde.

\section{Discussão}

Os resultados das revisões sistemáticas mostraram benefícios como redução na prescrição $^{6,17,30,31,34,35}$ e no uso de antibióticos ${ }^{6,25,28,29,32,33,35}$, redução no tempo de tratamento ${ }^{29}$ e melhora na adesão às diretrizes ${ }^{6,34}$. Contudo, nota-se que estes são resultados de desfechos intermediários, $\mathrm{o}$ que mostra a necessidade de condução de estudos com foco na redução e controle de resistência microbiana.

Destacaram-se as intervenções educacionais (especialmente aquelas dirigidas a prescritores e pacientes, treinamento em habilidades de comunicação, auditoria clínica, feedback) com resultados positivos apresentados nos desfechos relatados e que também foram apontadas como facilitadores de implementação das estratégias de enfrentamento do problema. Além disso, os biomarcadores também indicaram efeito positivo, mas ainda são recursos de difícil acesso dependendo da realidade regional de nosso país.

Apesar desses achados positivos, os resultados devem ser vistos com cautela, devido à escassez de estudos que analisaram programas de stewardship em serviços pediátricos ${ }^{17,30}$ e de estudos realizados em países de baixa e média renda $^{26,32}$, além da variedade de intervenções e de cenários clínicos analisados ${ }^{30}$.

A diversidade de metodologias empregadas nos estudos incluídos (revisões sistemáticas, meta-etnografias e pesquisa primária quantitativa e qualitativa) também torna mais complexa a avaliação da qualidade, bem como extração, interpretação e apresentação dos resultados. Ressalta-se que a maioria das revisões sistemáticas apresentou resultado entre qualidade metodológica baixa e criticamente baixa, o que pode diminuir a confiança nas evidências encontradas. Além disso, as buscas por revisões sistemáticas, para elaborar a síntese de evidências, não foram específicas para APS, e é possível que isso tenha influenciado a recuperação dos estudos nas bases de dados.

A APS é onde ocorre o maior uso de antibióticos ${ }^{51}$, mesmo que muitos países tenham alcançado a redução dessas prescrições ${ }^{52}$. Os motivos das prescrições normalmente estão relacionados a suspeitas de infecções respiratórias ${ }^{52}$, infecções urinárias ${ }^{53}$ e a prescrições inadequadas de antibióticos realizadas pelo medo de complicações e para atender desejos dos pacientes ${ }^{51}$.

$\mathrm{O}$ uso de diretrizes pode tornar ótimo o uso de antimicrobianos, desde que considere a realidade dos serviços de saúde, adaptando as dire- 
trizes quando necessário, principalmente pelas peculiaridades que se identifica na APS ${ }^{54}$.

A adesão a protocolos e diretrizes em serviços de saúde, entretanto, é prejudicada pelo conhecimento empírico de profissionais de saúde, especialmente dos prescritores. Dessa forma, são necessários pesquisas adicionais para identificar fatores determinantes no comportamento do prescritor durante consultas clínicas a fim de encontrar estratégias efetivas para prescrição prudente de antibióticos de acordo com os aspectos biopsicossociais dos pacientes 9 . O estudo espanhol de Vazquez-Lago et al. ${ }^{51}$, por exemplo, acerca das opiniões e atitudes dos clínicos gerais em relação a antibióticos e resistência antimicrobiana confirmam os dados encontrados na literatura sobre medo e insegurança no ato de prescrever.

Outro desafio na APS são as prescrições de antibióticos para pacientes pediátricos, comumente dispensadas em maior volume nesses serviços em comparação à população adulta ${ }^{1,9}$.

Estudos realizados em países de baixa renda salientam que a disponibilidade de medicamentos de segunda linha e o acesso reduzido a assistência médica podem aumentar a morbimortalidade por infecções resistentes a antibióticos ${ }^{1}$, também relatado no diálogo deliberativo como um obstáculo vivenciado por gestores de saúde em nosso país.
A APS é um campo bastante fértil para a adoção de estratégias voltadas à redução do consumo e prescrição de antimicrobianos. Dificilmente em outro nível de atenção é possível acessar o usuário ou seu cuidador, e intervenções com inclusão desses atores podem representar mudanças para além do período de tratamento. Também é o local onde toda equipe de profissionais de saúde pode ter maior protagonismo na atenção ao usuário e se beneficiar, como equipe, das estratégias implementadas. No entanto, a resistência às mudanças pode ser um grande obstáculo para a implementação bem-sucedida das intervenções efetivas. Há recomendações nacionais para prevenção da disseminação de microrganismos multirresistentes em diversos serviços de saúde, mas é escassa para a APS. Contudo, devido à falta de informações sobre gerenciamento de resistência microbiana nesse tipo de serviço, como por exemplo no manejo de pacientes com úlceras venosas, nota-se a importância de políticas públicas nesse nível de atenção $0^{55}$.

Apesar da potencialidade das intervenções analisadas nas revisões sistemáticas para reduzir o consumo e/ou a prescrição de antimicrobianos, claramente há necessidade de um plano de implementação, avaliação e monitoramento para que os benefícios sejam alcançados.

\section{Colaboradores}

JRA Bonfim contribuiu com a concepção teórica. BC Araújo e RC Melo contribuíram no processo de seleção, extração dos estudos, confecção do relatório e elaboração do manuscrito. MC Bortoli contribuiu na revisão do manuscrito. TS Toma coordenou e revisou o relatório e o manuscrito.

\section{Agradecimentos}

Bibliotecárias da Biblioteca da Faculdade de Medicina da Universidade de Campinas, por ajudar na estratégia de busca e fornecer os artigos de acesso restrito. 


\section{Referências}

1. Bryce A, Hay AD, Lane IF, Thornton H V, Wootton $\mathrm{M}$, Costelloe C. Global prevalence of antibiotic resistance in paediatric urinary tract infections caused by Escherichia coli and association with routine use of antibiotics in primary care: systematic review and meta-analysis. BMJ 2016 15; 352:i939.

2. Dreser A, Wirtz VJ, Corbett KK, Echániz G. Uso de antibióticos en México: Revisión de problemas y políticas. Salud Publica Mex 2008; 50(Supl. 4):S480-S487.

3. Mcewen SA, Collignon PJ. Antimicrobial Resistance: A One Health Perspective. Microbiol Spectr 2017; 6(2):1-26.

4. O'neill J. Antimicrobials in Agriculture and the Environment: Reducing Unnecessary Use and Waste the Review on Antimicrobial Resistance [Internet]. 2015 [cited 2019 nov 1]. Available from: https://amr-review.org/sites/default/files/Antimicrobials $\% 20$ in $\% 20$ agriculture $\% 20$ and $\% 20$ the $\% 20$ environment $\% 20$ -\%20Reducing\%20unnecessary\%20use $\% 20$ and $\% 20$ waste.pdf.

5. Klein EY, Van Boeckel TP, Martinez EM, Pant S, Gandra S, Levin SA, Goossens H, Laxminarayan R. Global increase and geographic convergence in antibiotic consumption between 2000 and 2015. Proc Natl Acad Sci USA 2018; 115(15):E3463-E3470.

6. Keller SC, Tamma PD, Cosgrove SE, Miller MA, Sateia H, Szymczak J, Gurses AP, Linder JA. Ambulatory antibiotic stewardship through a human factors engineering approach: A systematic review. J Am Board Fam Med 2018; 31(3):417-430.

7. De Waele JJ, Akova M, Antonelli M, Canton R, Carlet J, De Backer D, Dimopoulos G, Garnacho-Montero J, Kesecioglu J, Lipman J, Mer M, Paiva J-A, Poljak M, Roberts JA, Bano JR, Timsit J-F, Zahar J-R, Bassetti M. Antimicrobial resistance and antibiotic stewardship programs in the ICU: insistence and persistence in the fight against resistance. A position statement from ESICM/ESCMID/WAAAR round table on multi-drug resistance. Intensive Care Med 2018; 44(2):189-196.

8. Avoiding antibacterial overuse in primary care Reducing excessive. Drug Ther Bull 2007; 45:25-29.

9. Thompson W, Tonkin-Crine S, Pavitt SH, McEachan RRC, Douglas GVA, Aggarwal VR, Sandoe JAT. Factors associated with antibiotic prescribing for adults with acute conditions: an umbrella review across primary care and a systematic review focusing on primary dental care. J Antimicrob Chemother 2019; 74(8):2139-2152.

10. Krockow EM, Colman AM, Chattoe-Brown E, Jenkins DR, Perera N, Mehtar S, Tarrant C. Balancing the risks to individual and society: a systematic review and synthesis of qualitative research on antibiotic prescribing behaviour in hospitals. J Hosp Infect 2019; 101(4):428439.

11. Pan American Health Organization (PAHO). Florida International University. Recommendations for Implementing Antimicrobial Stewardship Programs in Latin America and the Caribbean: Manual for Public Health Decision-Makers [Internet]. Washington, D.C.: PAHO; 2018 [cited 2019 nov 1]. Available from: http://iris.paho.org/xmlui/handle/123456789/49645.
12. Lima HK, Carvalho HE, Sousa AF, Moura ME, Andrade D, Valle AR. Distribuição e custo de antimicrobianos na Atenção Primária. Acta Paul Enferm 2018; 31(1):95-101.

13. Organização Panamericana de Saúde (OPAS). OMS publica lista de bactérias para as quais se necessitam novos antibióticos urgentemente [Internet]. OPAS; 2017 [acessado 2019 nov 1]. Disponível em: https:// www.paho.org/bra/index.php?option=com_content\&view=article\&id=5357:oms-publica-lista-de-bacterias-para-as-quais-se-necessitam-novos-antibioticos-urgentemente\&Itemid $=812$.

14. World Health Organization (WHO). Antimicrobial Resistance: A Manual for developing national action plans [Internet] WHO; 2017 [cited 2020 maio 20]. Available from: http://apps.who.int/iris/bitstream/ handle/10665/204470/9789241549530_eng.pdf?sequence.

15. World Health Organization (WHO). Global antimicrobial resistance surveillance system (GLASS) report: early implementation 2016-2017 [Internet]. WHO; 2017 [cited 2020 maio 20]. Available from: http:// apps.who.int/iris/bitstream/handle/10665/259744/ 9789241513449-eng.pdf?sequence $=$.

16. Agência Nacional de Vigilância Sanitária (ANVISA). Plano Nacional paraaPrevençãoeo ControledaResistência Microbiana nos Serviços de Saúde [Internet]. ANVISA; 2017 [acessado 2019 nov 1]. Disponível em: http:// portal.anvisa.gov.br/documents/33852/271855/ Plano+Nacional+para $+\mathrm{a}+$ Preven $\% \mathrm{C} 3 \% \mathrm{~A} 7 \%$ $\mathrm{C} 3 \% \mathrm{~A} 3 \mathrm{O}+\mathrm{e}+\mathrm{o}+$ Controle $+\mathrm{da}+$ Resist $\% \mathrm{C} 3 \% \mathrm{AAncia}+-$ Microbiana+nos+Servi\%C3\%A7os+de+Sa\%C3\%BA de/9d9f63f3-592b-4fe1-8ff2-e035fcc0f31d.

17. Drekonja DM, Filice GA, Greer N, Olson A, MacDonald R, Rutks I, Wilt TJ. Antimicrobial stewardship in outpatient settings: A systematic review. Infect Control Hosp Epidemiol 2015; 36(2):142-152.

18. Lavis JN, Oxman AD, Lewin S, Fretheim A. Ferramentas SUPPORT para a elaboração de políticas de saúde baseadas em evidências (STP). Heal Res Policy Syst 2009; 7(1):1478-4505.

19. Toma TS, Tesser TR, Setti C, Bortoli MC. Síntese de evidências para políticas de saúde. In: Toma TS, Preira TV, Vanni T, Barreto JOM, organizadores. Avaliação de tecnologias de saúde \& políticas informadas por evidências. São Paulo: Instituto de Saúde; 2017. p. 183208.

20. Shea BJ, Reeves BC, Wells G, Thuku M, Hamel C, Moran J, Moher D, Tugwell P, Welch V, Kristjansson E, Henry DA. AMSTAR 2: a critical appraisal tool for systematic reviews that include randomised or non -randomised studies of healthcare interventions, or both. BMJ 2017; 358: 4008 .

21. Mulvale G, Chodos H, Bartram M, MacKinnon MP, Abud M. Engaging civil society through deliberative dialogue to create the first Mental Health Strategy for Canada: Changing Directions, Changing Lives. Soc Sci Med 2014; 123:262-268.

22. Nabyonga-Orem J, Ousman K, Estrelli Y, Rene AK, Yakouba Z, Gebrikidane M, Mamoud D, Kwamie A. Perspectives on health policy dialogue: definition, perceived importance and coordination. BMC Health Serv Res 2016; 16(Supl. 4):218. 
23. Melo RC, Araújo BC, Bortoli MC, Toma TS. Gestão das intervenções de prevenção e controle da resistência a antimicrobianos em hospitais: revisão de evidências. Rev Panam Salud Publica 2020; 44:e35.

24. Moher D, Liberati A, Tetzlaff J, Altman DG, The PRISMA Group. Preferred Reporting Items for Systematic Reviews and Meta-Analyses: The PRISMA Statement. PLoS Med 2009; 6(7):e1000097.

25. Aabenhus R, Jensen J-US, Jorgensen KJ, Hrobjartsson A, Bjerrum L. Biomarkers as point-of-care tests to guide prescription of antibiotics in patients with acute respiratory infections in primary care. Cochrane Database Syst Rev 2014; (11):CD010130.

26. Coxeter P, Del Mar CB, McGregor L, Beller EM, Hoffmann TC. Interventions to facilitate shared decision making to address antibiotic use for acute respiratory infections in primary care. Cochrane Database Syst Rev 2015; (11):CD010907.

27. Löffler C, Böhmer F. The effect of interventions aiming to optimise the prescription of antibiotics in dental care - A systematic review. PLoS One 2017; 12(11):1-23.

28. O'Sullivan JW, Harvey RT, Glasziou PP, McCullough A. Written information for patients (or parents of child patients) to reduce the use of antibiotics for acute upper respiratory tract infections in primary care. Cochrane Database Syst Rev 2016; 11:CD011360.

29. Schuetz P, Wirz Y, Sager R, Christ-Crain M, Stolz D, Tamm M, Bouadma L, Luyt CE, Wolff M, Chastre J, Tubach F, Kristoffersen KB, Burkhardt O, Welte T, Schroeder S, Nobre V, Wei L, Bucher HC, Bhatnagar $\mathrm{N}$, Annane D, Reinhart K, Branche A, Damas P, Nijsten M, Lange DW, Deliberato RO, Lima SS, Maravić -Stojković V, Verduri A, Cao B, Shehabi Y, Beishuizen A, Jensen J-US, Corti C, Van Oers JA, Falsey AR, Jong E, Oliveira CF, Beghe B, Briel M, Mueller B. Procalcitonin to initiate or discontinue antibiotics in acute respiratory tract infections. Cochrane Database Syst Rev 2017; 10:CD007498.

30. Arnold SR, Straus SE. Interventions to improve antibiotic prescribing practices in ambulatory care (Review). Cochrane Database Syst Rev 2005; 2005(4): CD003539.

31. Hu Y, Walley J, Chou R, Tucker JD, Harwell JI, Wu $X$, Yin J, Zou G, Wei X. Interventions to reduce childhood antibiotic prescribing for upper respiratory infections: Systematic review and meta-analysis. J Epidemiol Community Health 2016; 70(12):1162-1170.

32. Andrews T, Thompson M, Buckley DI, Heneghan C, Deyo R, Redmond N, Lucas PJ, Blair PS, Hay AD. Interventions to influence consulting and antibiotic use for acute respiratory tract infections in children: a systematic review and meta-analysis. PLoS One 2012; 7(1):e30334.

33. Patel SJ, Larson EL, Kubin CJ, Saiman L. A review of antimicrobial control strategies in hospitalized and ambulatory pediatric populations. Pediatr Infect Dis J 2007; 26(6):531-537.

34. Ivanovska V, Holloway KA. Interventions to improve antibiotic prescribing in upper middle income countries: A systematic review of the literature 1990-2009. Maced J Med Sci 2013; 6(1):84-91.
35. Roque F, Herdeiro MT, Soares S, Teixeira Rodrigues A Breitenfeld L, Figueiras A. Educational interventions to improve prescription and dispensing of antibiotics: a systematic review. BMC Public Health 2014; 14:1276.

36. Bal AM, Gould IM. Antibiotic stewardship: Overcoming implementation barriers. Curr Opin Infect Dis 2011; 24(4):357-362.

37. Dobson EL, Klepser ME, Pogue JM, Labreche MJ, Adams AJ, Gauthier TP, Turner RB, Su CP, Jacobs DM, Suda KJ, SIDP Community Pharmacy Antimicrobial Stewardship Task Force. Outpatient antibiotic stewardship: Interventions and opportunities. $J$ Am Pharm Assoc 2017; 57(4):464-473.

38. Bassetti M, Poulakou G, Ruppe E, Bouza E, Van Hal SJ, Brink A. Antimicrobial resistance in the next 30 years, humankind, bugs and drugs: a visionary approach. Intensive Care Med 2017; 43(10):1464-1475.

39. Özgenç O. Methodology in improving antibiotic implementation policies. World J Methodol 2016; 6(2):143.

40. Tamma PD, Cosgrove SE. Antimicrobial stewardship. Infect Dis Clin North Am 2011; 25(1):245-260.

41. D'Agata EMC. Antimicrobial use and stewardship programs among dialysis centers. Semin Dial 2013; 26(4):457-464.

42. Cunha CB, D'Agata EMC. Implementing an antimicrobial stewardship program in out-patient dialysis units. Curr Opin Nephrol Hypertens 2016; 25(6):551555.

43. World Health Organisation (WHO). Turning Plans into Action for Antimicrobial Resistance (AMR) Working Paper 2.0: Implementation and Coordination [Internet]. 2019 [cited 2019 nov 1]. Available from: https://www.who.int/antimicrobial-resistance/publications/AMR-Turning-plans-into-action-working -paper-march-2019.pdf,

44. Van Buul LW, Sikkens JJ, Van Agtmael MA, Kramer MHH, Van der Steen JT, Hertogh CMPM. Participatory action research in antimicrobial stewardship: A novel approach to improving antimicrobial prescribing in hospitals and long-term care facilities. J Antimicrob Chemother 2014; 69(7):1734-1741.

45. Cox JA, Vlieghe E, Mendelson M, Wertheim H, Ndegwa L, Villegas MV, Gould I, Hara GL. Antibiotic stewardship in low- and middle-income countries: the same but different? Clin Microbiol Infect 2017; 23(11):812-818.

46. Allerberger F, Lechner A, Wechsler-Fördös A, Gareis R. Optimization of antibiotic use in hospitals - Antimicrobial stewardship and the EU Project ABS International. Chemotherapy 2008; 54(4):260-267.

47. Allerberger F, Frank A, Gareis R, et al. Antibiotic stewardship through the EU project "ABS International”. Wien Klin Wochenschr 2008; 120(9-10):256-263.

48. Bishop BM. Antimicrobial Stewardship in the Emergency Department: Challenges, Opportunities, and a Call to Action for Pharmacists. J Pharm Pract 2016; 29(6):556-563.

49. Warreman EB, Lambregts MMC, Wouters RHP, Visser LG, Staats H, van Dijk E, Boer MGJ. Determinants of in-hospital antibiotic prescription behaviour: a systematic review and formation of a comprehensive framework. Clin Microbiol Infect 2019; 25(5):538-545. 
50. Schuetz P, Bolliger R, Merker M, Christ-Crain M, Stolz D, Tamm M, Luyt CE, Wolff M, Schroeder S, Nobre V, Reinhart K, Branche A, Damas P, Nijsten M, Deliberato RO, Verduri A, Beghé B, Cao B, Shehabi Y, Jensen J-US, Beishuizen A, Jong E, Briel M, Welte T, Mueller B. Procalcitonin-guided antibiotic therapy algorithms for different types of acute respiratory infections based on previous trials. Expert Rev Anti Infect Ther 2018; 16(7):555-564.

51. Vazquez-Lago JM, Lopez-Vazquez P, López-Durán A, Taracido-Trunk M, Figueiras A. Attitudes of primary care physicians to the prescribing of antibiotics and antimicrobial resistance: a qualitative study from Spain. Family Practice 2012; 29(3):352-360.

52. Costelloe C, Metcalfe C, Lovering A, Mant D, Hay Alastair D. Effect of antibiotic prescribing in primary care on antimicrobial resistance in individual patients: systematic review and meta-analysis. $B M J$ 2010; 340:c2096.

53. Wawrysiuk S, Naber K, Rechberger T, Miotla P. Prevention and treatment of uncomplicated lower urinary tract infections in the era of increasing antimicrobial resistance-non-antibiotic approaches: a systemic review. Arch Gynecol Obstet 2019; 300(4):821-828.

54. Tavares NUL, Bertoldi AD, Muccillo-Baisch AL. Prescrição de antimicrobianos em unidades de saúde da família no Sul do Brasil. Cad Saude Publica 2008; 24(8):1791-1800.

55. Santos SDLVD, Martins MA, Vasconcelos LSNDO, Lima ABM, Malaquias SG, Bachion MM. Bastonetes Gram-negativos em úlceras venosas e implicações para o atendimento de enfermagem na atenção primária. Rev Eletr Enferm 2014; 16(2):370-377.

Artigo apresentado em 24/03/2020

Aprovado em 18/11/2020

Versão final apresentada em 20/11/2020

Editores-chefes: Romeu Gomes, Antônio Augusto Moura da Silva 